\title{
Strategies for Intravenous Fluid Resuscitation in Trauma Patients
}

\author{
Robert Wise $^{1,2,3} \cdot$ Michael Faurie $^{4}$ Manu L. N. G. Malbrain ${ }^{5}$ Eric Hodgson ${ }^{3,6}$
}

Published online: 5 January 2017

(c) The Author(s) 2017. This article is published with open access at Springerlink.com

\begin{abstract}
Intravenous fluid management of trauma patients is fraught with complex decisions that are often complicated by coagulopathy and blood loss. This review discusses the fluid management in trauma patients from the perspective of the developing world. In addition, the article describes an approach to specific circumstances in trauma fluid decision-making and provides recommendations for the resource-limited environment.
\end{abstract}

\section{Initial resuscitation fluid management}

\section{Background}

Fluid resuscitation of trauma patients has been an ongoing challenge, constantly reviewed and debated, resulting in recommendations changing for the use of crystalloids/colloids/packed red blood cells/warm fresh whole blood and clotting factors. Other challenges, such as limited resources, impact the practitioners' choice of fluid-the best fluid

Robert Wise

Robert.Wise@kznhealth.gov.za

1 Pietermaritzburg Metropolitan Department of Anaesthetics, Critical Care and Pain Management, Pietermaritzburg, South Africa

2 Head Clinical Unit, Critical Care, Edendale Hospital, Pietermaritzburg, South Africa

3 Discipline of Anaesthesia and Critical Care, School of Clinical Medicine, College of Health Sciences, Nelson R Mandela School of Medicine, University of KwaZulu-Natal, Durban, South Africa

4 Trauma and Critical Care Fellow, Inkosi Albert Luthuli Central Hospital, Durban, South Africa

5 Ziekenhuis Netwerk Antwerpen, ZNA Stuivenberg, Lange Beeldekensstraat 267, 2060 Antwerpen 6, Belgium

6 Inkosi Albert Luthuli Central Hospital, Durban, South Africa available does not always equate to the best fluid for the patient, especially where long transfers and no blood availability are concerned. These decisions and management strategies appear relevant for further discussion and research, as this fluid resuscitation attempts to provide adequate organ perfusion and oxygen delivery in a system compromised by the physiological consequences of injury. Several questions have arisen from this topic: Which fluid is best, how much should be given, and do specific injuries call for different strategies (for example, penetrating vs. blunt trauma)? Achieving balance in the resuscitation period is challenging, particularly the volume administered. More fluid is not always better, in fact, quite the contrary [1-3]. Much of the literature on fluid resuscitation focuses on critically ill patients with sepsis, or elective perioperative patients [4-7]. Small cohorts of trauma patients can be found in the larger studies, but it should be remembered that most of these data include patients from the ICU setting $[4,5]$. Extrapolation of data to the initial resuscitation phase of the trauma patient is not possible $[8,9]$. This article emphasizes different types of fluid available, when they should be used, and recommendations on how to tailor fluid resuscitation through monitoring techniques. The goals of improving physiology, restoring or maintaining normothermia and minimizing coagulopathy should be considered paramount throughout the discussion. 


\section{Penetrating versus blunt injury versus head injuries}

There are three distinct groups of trauma patients, but often there is an overlap. Most commonly encountered is the combination of blunt trauma and head injury associated with motor vehicle collisions. While the general Advanced Trauma Life Support (ATLS) management approach to the three groups remains similar, fluid therapy strategies differ. The literature suggests patients with penetrating injuries, particularly to the thoracoabdominal region, have better outcomes with restrictive clear fluid resuscitation policies, permitting a systolic blood pressure (BP) between 60 and $70 \mathrm{mmHg}$ until the patient can be taken to the operating theater [10]. Once hemorrhage has been controlled in theater and blood products are available, higher blood pressure values may be targeted. There have been no large trials comparing restrictive and liberal fluid strategies in the context of blunt injury. However, a restrictive policy is acceptable with slower infusions favored over rapid boluses [10]. A slightly higher systolic blood pressure of $80-90 \mathrm{mmHg}$ is permitted, again, until control in theater is achieved and blood products are available. This restrictive policy is thought to minimize intra-abdominal bleeding while maintaining adequate organ perfusion and reducing the risk of intra-abdominal hypertension and complications mentioned previously. It should be remembered, however, that clinical scenarios are often complicated, and blood pressure goals should be individualized according to patient physiology, comorbidities and physiological compensation to shock during the time of resuscitation.

The exception to the guidelines above is the polytrauma patient (blunt or penetrating) with traumatic brain injury (TBI). In order to preserve adequate cerebral perfusion pressure and prevent secondary brain injury, one needs to target a mean arterial pressure (MAP) of greater than $80 \mathrm{mmHg}$ (a cerebral perfusion pressure of approximately $60 \mathrm{mmHg}$ ) [10].

\section{Clear fluid resuscitation}

The ongoing debate as to which group of fluid (synthetic colloid or crystalloid solutions [3]) is best to use in the resuscitation phase of trauma patients remains unanswered with large studies showing little, if any benefit of hydroxyethyl starch 130/0.4 [11] over the traditionally used crystalloids. The CRISTAL trial did identify a potential mortality benefit in a heterogeneous hypovolemic patient cohort resuscitated with a variety of colloid solutions compared to crystalloid solutions. However, several limitations identified by the authors limits applicability: the lack of renal injury and potential 90-day outcome benefit, deserve further research [7]. When reviewing the available literature, in several trials recruitment and consent requirements resulted in the comparison of fluids commencing after the initial resuscitation phase, resulting in interpretation difficulties of outcome benefit in trauma patients $[4,5,7,12]$. These studies do, however, demonstrate a trend toward less synthetic colloid fluid required to achieve hemodynamic goals compared to crystalloids with a ratio (volume of colloid to crystalloid that results in similar physiological effects) varying between 1:1.1 and 1:1.6 (colloids:crystalloids) [4, 5, 7, 12]. This ratio is smaller than previously thought (ATLS teaches a ratio of 1:3), and significance in subgroups of patients is yet to be determined. Concerns still exist about the adverse effects of hydroxyethyl starch 130/0.4 on renal function and coagulopathy although crystalloid fluids are not without complications [7]. Further studies need to be done comparing these crystalloids and colloids in the initial resuscitation phase of trauma patients. A further concern is the chloride load administered in these fluids and the potential contribution toward acidosis and renal injury [13]. The resultant hyperchloremic metabolic acidosis may have negative consequences. The meta-analysis by Krajewski et al. [13] showed a significant association between high chloride content fluids and acute kidney injury, blood transfusion volume and mechanical ventilation time. Mortality was unaffected in this population of perioperative patients. Despite this, $0.9 \%$ saline remains widely used as a resuscitation fluid and remains the fluid of choice for patients with brain injury, hyponatremia and metabolic alkalosis. Balanced salt solutions (solutions with a physiological $\mathrm{pH}$ and isotonic electrolyte concentration), being more physiological in nature, are being used more frequently, showing a trend toward less harm than $0.9 \%$ sodium chloridewhether in isolation or as a medium carrying a colloid [14]. Balanced salt solutions closely resemble human plasma and thus have a lower sodium and chloride content than $0.9 \%$ saline with the addition of a buffer such as acetate or lactate. These fluids (e.g., Ringer's lactate, Hartmann's solution) have minimal effects on $\mathrm{pH}$ but are hypotonic, so can exacerbate edema, particularly cerebral edema in the injured brain. In addition, when using Ringer's lactate solution, consideration should be given to the potential interaction between citrate found in stored blood and bicarbonate, explaining why $0.9 \%$ saline is still a commonly used resuscitation fluid in trauma patients, despite the high chloride load [15]. The concerns regarding the inflammatory effects from Ringers lactate infusions, demonstrated in animal models, have not been demonstrated to influence outcomes in human studies. Of greater concern are the negative consequences of hyperchloremic metabolic acidosis. There are no large randomized control trials demonstrating a mortality benefit for $0.9 \%$ saline over balanced solutions. Currently, saline is preferred in braininjured patients and balanced solutions are preferred in 
patients who are already acidotic. Although only in an porcine model, resuscitation after severe hemorrhage with $0.9 \%$ saline was inferior to ringers lactate due to vasodilatory effect, and risks of metabolic acidosis and hyperkalemia [16]. In elective neurosurgical patients, lactated ringers also proved better than $0.9 \%$ saline in terms of electrolyte management (particularly sodium and chloride) and acid-base balance [17].

In the resource-limited environment, the use of cheaper crystalloid solutions is still recommended due to lack of data showing significant outcome benefits of more expensive synthetic colloids. A selection of different crystalloid solutions is often not available in resource-limited settings, making the available solution the only and best choice. In a Cochrane review, the use of hypertonic saline for the resuscitation of trauma victims has failed to show any benefit over isotonic or near-isotonic crystalloids and two adequately powered trials investigating mortality as an endpoint were halted early due to futility. Controversy, however, still continues fueled by animal studies demonstrating benefits that have not been reflected as outcome benefits in human studies [18]. Heterogeneous populations and methodological differences between studies make the interpretation of evidence difficult. Hypertonic saline may have a role when used in the head-injured patient as a bridge to neurosurgery [19].

Several trials show either no benefit, or in some cases worse outcomes, with albumin thus making this solution not recommended in the resuscitation of trauma patients [20-22].

The physiological impact of the volume of fluid infused may be as, or even more important than the type selected [2, 23-26]. Excessive fluid results in a dilutional coagulopathy and diffuse tissue edema. This negatively impacts organ function at both a macroscopic and cellular level by increasing the distance over which electrolytes, elements and oxygen have to move [3]. The consequence is worsening renal, hepatic and cardiac function as well as increasing volume of extra vascular lung water that worsens ventilation-perfusion mismatch. Abdominal hypertension/compartment syndrome may progress to a polycompartment syndrome [23, 26].

Therefore, until such time as blood and blood products are available, clear fluid resuscitation should be limited to only that which is necessary to maintain adequate organ perfusion. Several factors influence decisions at this point of the resuscitation. Trauma units with easy access to onsite blood and blood products should commence resuscitation of patients with massive blood loss with these products from the start. In environments where blood products are limited, the authors suggest judicious use of clear fluids to sustain organ perfusion while avoiding the negative effects of excess fluid. Response to fluid administration and determining the need for further fluid administration is discussed in the next section.

As defined by the Advanced Trauma Life Support course, classification of patients into those that respond to initial fluid resuscitation versus those that only transiently respond or do not respond at all is important. The response to intravenous fluid resuscitation is assessed using physiological markers of improvement such as blood pressure, heart rate, decreasing lactate and normalizing base deficit with adequate control of bleeding. Responders are considered those that demonstrate these physiological improvements, whereas transient responders show an initial improvement followed by further physiological deterioration. Non-responders are those that show continued physiological deterioration despite initial fluid resuscitation. The distinction requires vigilance and repeated clinical assessments to identify those patients with re-bleeding, or ongoing bleeding, and initiation of blood product resuscitation together with surgical intervention. What may be regarded as acceptable physiological parameters will vary depending on many factors including the age, underlying medication and comorbidities of the patient.

See Fig. 1 for a guide to initial fluid management in trauma patients.

\section{Blood and blood products}

The goal of resuscitation is to achieve adequate tissue perfusion and oxygenation while correcting any coagulopathy. Packed red blood cells, and to some extent hemoglobin-based oxygen carriers (HBOCs), help to achieve the former while component therapy attempts to deal with the coagulopathy. Whole blood may achieve both objectives [27]. Currently, there is no consensus definition for a massive blood transfusion [28]; however, recommendations for the concept of massive transfusions suggest plasma:platelets:red blood cells in a ratio of $1: 1: 1$ or $1: 1: 2$ [29]. This objective is seldom achieved due to limited access and supply of blood products in developing countries, where a 1:1:2 ratio is more easily achieved. An alternative to the use of this ratio is the use of warm fresh whole blood that has a higher hematocrit, more platelets and higher percentage of functional clotting factors per unit volume when compared to component therapy [27]. The concerns about its use, however, include a slightly higher sepsis rate, possible increased risk of acute kidney injury, as well as the risk of transfusion-related immunomodulation (TRIM), although this is minimized by the use of leukodepletion filters [27]. Several trials exist comparing the use of component therapy [30] to warm fresh whole blood, but many did not make this direct comparison, but rather a combination of warm fresh whole blood with 


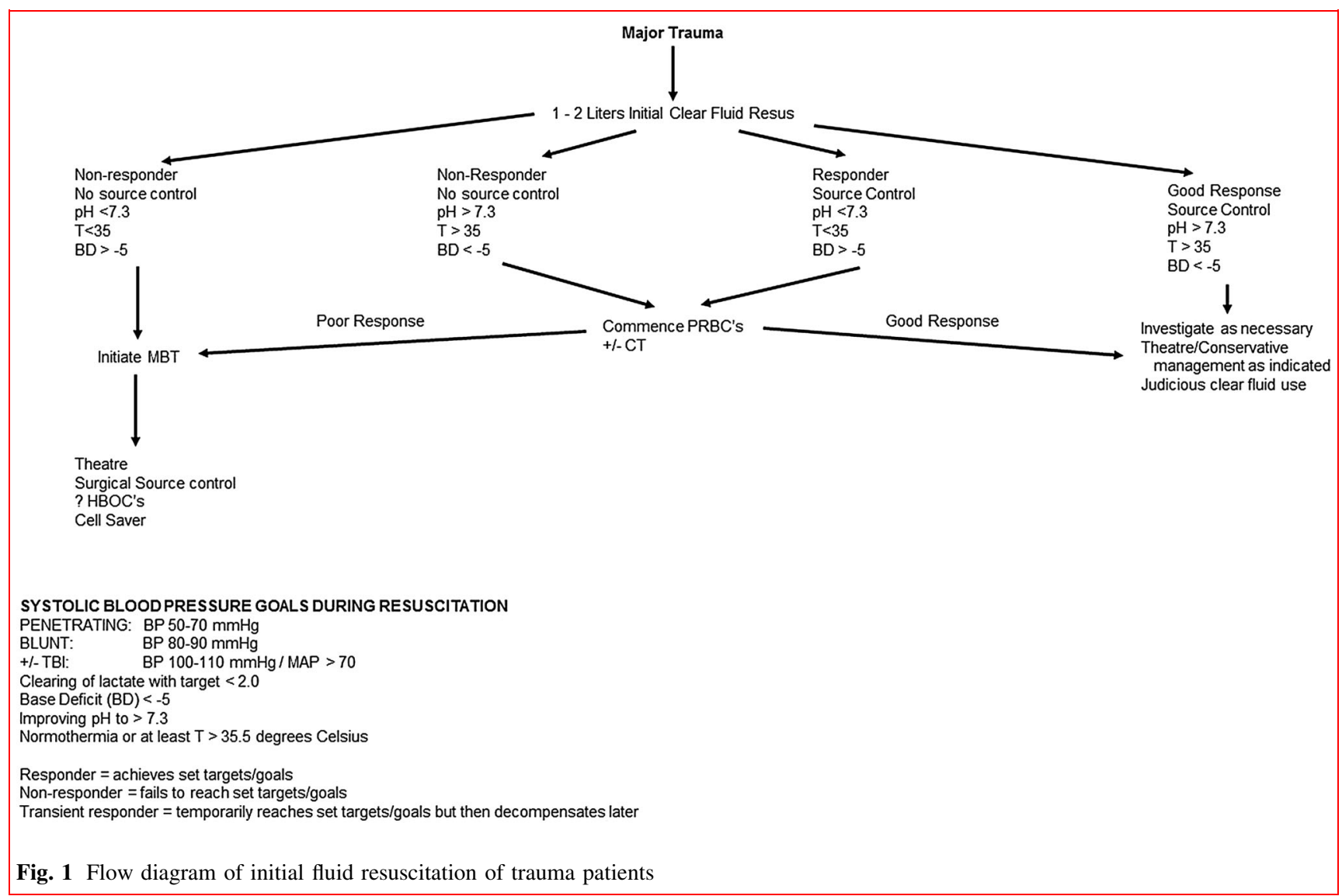

packed red blood cells and plasma, making the comparison difficult. Both 24-h and 30-day survival were higher in the warm fresh whole blood/red blood cell/plasma group compared with the component therapy only group, but further trials comparing these groups are necessary [27]. Whole blood also causes less dilutional effect and offers a higher concentration of fibrinogen than component therapy. The current preference for massive transfusion is trending toward the use of whole blood; however, if this is not readily available, as in the resource constrained environments, component therapy with a 1:1:1 ratio should be utilized [27-29, 31, 32].

\section{Hemoglobin solutions}

Modified hemoglobin solutions are not substitutes for blood as they do not possess the metabolic functions of erythrocytes. They act purely as oxygen carriers [33]. Large-scale investigation and experimentation in this field has occurred, with uses not only limited to trauma patients but also general surgical patients, oncology patients and Jehovah's Witnesses suffering from severe anemia resulting from various reasons [33]. The only product that was registered for use, in South Africa and later Russia, was
Hemopure $^{(\mathrm{R})}$ (HbO2 Therapeutics LLC, South Africa), used in isolation as well as in combination with blood products, or as a bridge to blood transfusion [34, 35]. Hemoglobin solutions do not only help oxygen transportation, but also enhance the release of oxygen from native hemoglobin at tissue level with some of them having a positive inotropic effect that may be useful in shocked trauma patients. This positive inotropy is related to the rate of administration and if given slowly is negligible [33, 34]. Serious adverse events (SAEs) were rare with the most serious being fluid overload. A recent review of the literature highlights several flaws in Natanson's previous metaanalysis suggesting that although a small minority of HBOCs have had serious adverse events (myocardial ischemia, cerebrovascular accidents), this cannot be extrapolated as a class effect due to the vast differences among HBOCs with respect to their structure, hemoglobin concentration and nitric oxide scavenging effects. In light of this, there is renewed interest in the use of HBOCs, especially the Hemopure ${ }^{(\mathrm{R})}(\mathrm{HbO} 2$ Therapeutics LLC, South Africa) compound, which to date has had the most success with fewest serious adverse events [36]. Further studies are required before these therapies become widely used. 
Table 1 Thromboelastometry interpretation and action guide

\begin{tabular}{lll}
\hline Laboratory value & Interpretation & Blood product transfusion \\
\hline$R$ time $<4 \mathrm{~min}$ & Enzymatic hypercoagulability & Do not treat if bleeding \\
$R$ time $>11 \mathrm{~min}$ & Low clotting factors & FDP/FFP's and RBC's \\
Alpha angle $>45$ degrees & Low fibrinogen levels & Cryoprecipitate/fibrinogen/platelets \\
$\mathrm{MA}<54 \mathrm{~mm}$ & Low platelet function & Platelets/cryoprecipitate/fibrinogen \\
$\mathrm{MA}>73 \mathrm{~mm}$ & Platelet hypercoagulability & Do not treat if bleeding \\
$\mathrm{LY} 30>3 \%$ & Primary fibrinolysis & Tranexamic acid $1 \mathrm{~g} \mathrm{IV} \mathrm{over} 10 \mathrm{~min}$ then $1 \mathrm{~g} / 250 \mathrm{ml} \mathrm{NS}$ over $8 \mathrm{~h}$ \\
$\mathrm{CI}<1.0$ & & \\
\hline
\end{tabular}

$F D P$ freeze-dried plasma, $F F P$ fresh-frozen plasma, $R B C$ red blood cell

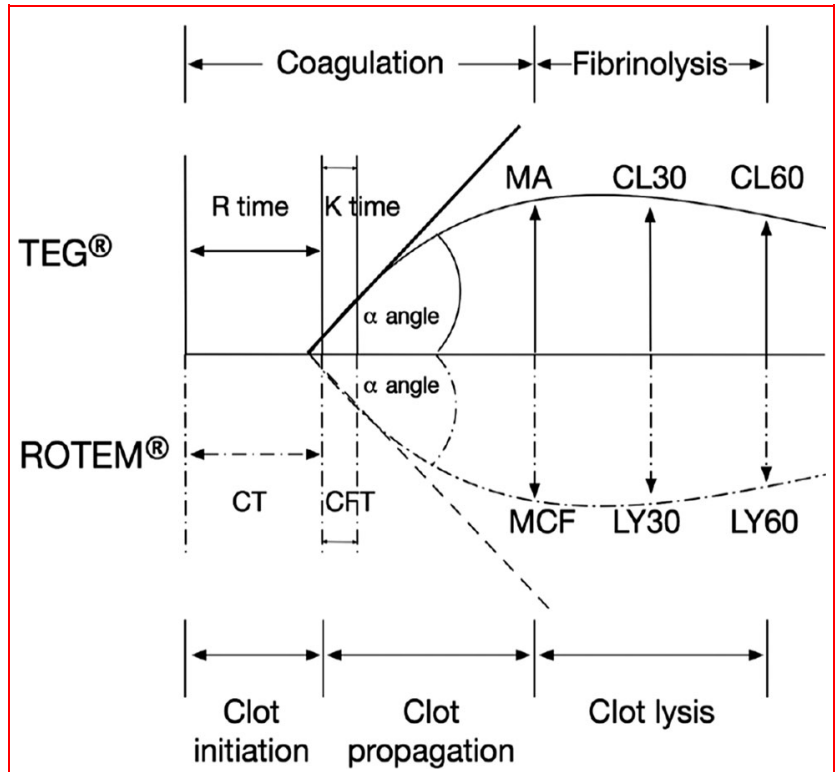

Fig. 2 Thromboelastometry-differences in measurement between ROTEM and thromboelastography [42]

\section{Monitoring coagulopathy}

Trauma-induced coagulopathy [37] is a relatively new concept, and the pathophysiology is still not completely understood. Traditionally, tests such as prothrombin/international normalized ratio [38] and partial thromboplastin time (PTT) were previously used to make this diagnosis. While these tests form the mainstay of trauma-induced coagulopathy testing in many centers, they only focus on the initial part of clot formation and not on the evolution of the clot or clot lysis. D-dimer and fibrinogen levels are used as surrogate markers of fibrinolysis and clotting factor consumption, respectively, but again these are non-specific when assessing coagulation in the injured patient [71]. Diagnosis of trauma-induced coagulopathy using these older assays is defined as: $\mathrm{PT}>18 \mathrm{~s}, \quad$ INR $>1.5$, PTT $>60 \mathrm{~s}$ or any of these values at a threshold of 1.5 times their reference value.
Monitoring coagulation in trauma-induced coagulopathy has been made easier with the use of point-of-care testing, namely viscoelastic assays. Recent evidence shows that it improves survival in patients requiring massive blood transfusions compared to those monitored by more traditional assays as described above [39]. While challenges may exist in introducing point-of-care testing into resource-limited settings, these systems would be ideal as they reduce dependency on traditional laboratory testing and allow for real-time feedback and goal-directed blood product use. Viscoelastic assays guide component replacement instead of resuscitating without reproducible biological guidance [40]. By initially assessing patients' thrombotic deficiencies and continuously re-assessing them following resuscitation with component therapy/whole blood, thromboelastometry guides resuscitation and potentially minimizes the use of allogeneic blood products resulting in reduced risk of transfusion-related side effects and minimizing costs [41]. Table 1 offers a guide to thromboelastometry interpretation and appropriate actions, but a more detailed description and management based on viscoelastic assays is beyond the scope of this review (Fig. 2).

Recommendations for current best practices:

1. Try and identify risk factors and priorities in trauma patients early (i.e., traumatic brain injury, penetrating injury, ongoing blood losses, compartment syndromes).

2. Consider early administration of blood products in a ratio of $1: 1: 1$ or $1: 1: 2$ if available.

3. In the absence of blood products, use clear fluid resuscitation. Preferably, a balanced salt solution should be used (such as Ringer's lactate or PlasmaLyte); however, care should be taken not to mix this fluid with blood transfusions.

4. When using clear fluids in resuscitation, vigilance is required to provide only that fluid which is necessary to maintain perfusion. Excessive clear fluid administration has negative consequences such as dilution of 
coagulation factors, tissue edema, hyperchloremic metabolic acidosis and organ dysfunction.

5. Point-of-care testing should be used whenever possible to guide component therapy replacement to correct coagulopathy.

6. In rural LMIC or long-distance transfer situations with no access to blood, a synthetic colloid may be of benefit in reducing subsequent edema and bowel anastomotic breakdown.

\section{Post-resuscitation fluid management}

\section{Background}

The consequences of under- or over-resuscitation with intravenous fluids are both detrimental [43, 44]. Hypovolemia resulting in adrenergic stimulated vasoconstriction, hypervolemia and fluid overload, massive intravenous fluid administration causing large sodium loads, dilution of coagulation factors, and rapid administration of cold fluids all result in damage to the endothelial layer and glycocalyx, impairment of microcirculatory function and inappropriate fluid shifts. The decision when to transition from an initial resuscitation phase to a post-resuscitation period is critical to a successful outcome.

Following the initial resuscitation phase, physiological targets may change despite the overall objective of adequate tissue perfusion remaining the primary goal. The post-resuscitation period may be considered after:

- Hemostasis and correction of coagulopathy (ongoing blood product replacement no longer required) [45].

- Evidence of improving microcirculatory flow (for example, improving lactate and blood gas parameters) [45].

- Hemodynamic stability (systolic blood pressure $>100 \mathrm{mmHg}$ with a mean arterial blood pressure of $>65 \mathrm{mmHg}$ in most cases; no longer need for inotropic or vasopressor support; an improving pulse rate in the presence of appropriate analgesia).

At this stage, most patients will no longer be responsive to rapid fluid administration, with normalization of markers for volume status (barometric or volumetric preload indicators) and markers for fluid responsiveness (pulse pressure variation (PPV), stroke volume variation (SVV), passive leg raise (PLR) test) [46].

Importantly, in certain circumstances where patients remain fluid responsive, curtailment of ongoing fluid resuscitation may be necessary if the tolerance to further intravenous fluid is deemed detrimental to physiological processes (for example, abrupt increase in extravascular lung water; worsening intra-abdominal pressure or abdominal compartment syndrome; difficult ventilation) [44]. Alternative strategies should be considered including inotropic support guided by cardiac output monitoring, alternative fluid strategies, and planning for hemodialysis with net ultrafiltration.

The clinician should be aware of the different phases within fluid management according to the ROSE concept. After the initial resuscitation $(R)$ phase comes organ support $(O)$ and stabilization $(S)$, and finally evacuation $(E)$ of excess fluids may be needed in some patients (Table 2) [47].

These are broad guidelines, and exceptions do exist. Urine output has not been included in our recommendations due to the many influencing factors, although it may be one of the only parameters to measure, particularly in resource-limited settings. The renal response to hypovolemia is multifactorial and depends on a combination of renal blood flow, renal perfusion pressure and plasma oncotic pressure. The type of resuscitation fluid is important as it may influence oncotic pressure. In addition, the neurohormonal control of renal function may cloud the clinical picture and appropriate retention of water may be interpreted as renal dysfunction when this may be an appropriate physiological fluid conservation mechanism. As outlined by Peeters et al., previous studies have not found a correlation between urine output and invasively derived physiological variables. Also, several studies have pointed to the inaccuracy of using urine output as a resuscitation target and its limitation in identifying fluid responders. This situation is complicated further in trauma patients in the presence of increased intra-abdominal pressure. The limitations of using urine output should be understood by attending physicians.

Fluids should be treated as drugs: Not only the type of fluid is important, but also the dose, the administration speed, the duration and de-escalation [48]. There are only three indications for giving fluids: resuscitation, replacement or maintenance [49].

\section{Maintenance fluid}

In providing maintenance fluids, care should be taken to avoid causing tissue edema. This requires limitation of crystalloid administration, which can only really be achieved in the post-resuscitation period. Crystalloid fluid administration is not without hazards. Excessive crystalloid administration is associated with edema of skin, abdominal organs (leading to abdominal compartment syndrome), kidneys (leading to renal compartment syndrome, contributing to acute renal failure) and heart (leading to myocardial dysfunction) [44]. The ideal concept would be to use a strategy where the fluid stays intravascular and 
Table 2 ROSE concept (adapted from Malbrain et al. with permission) [40]

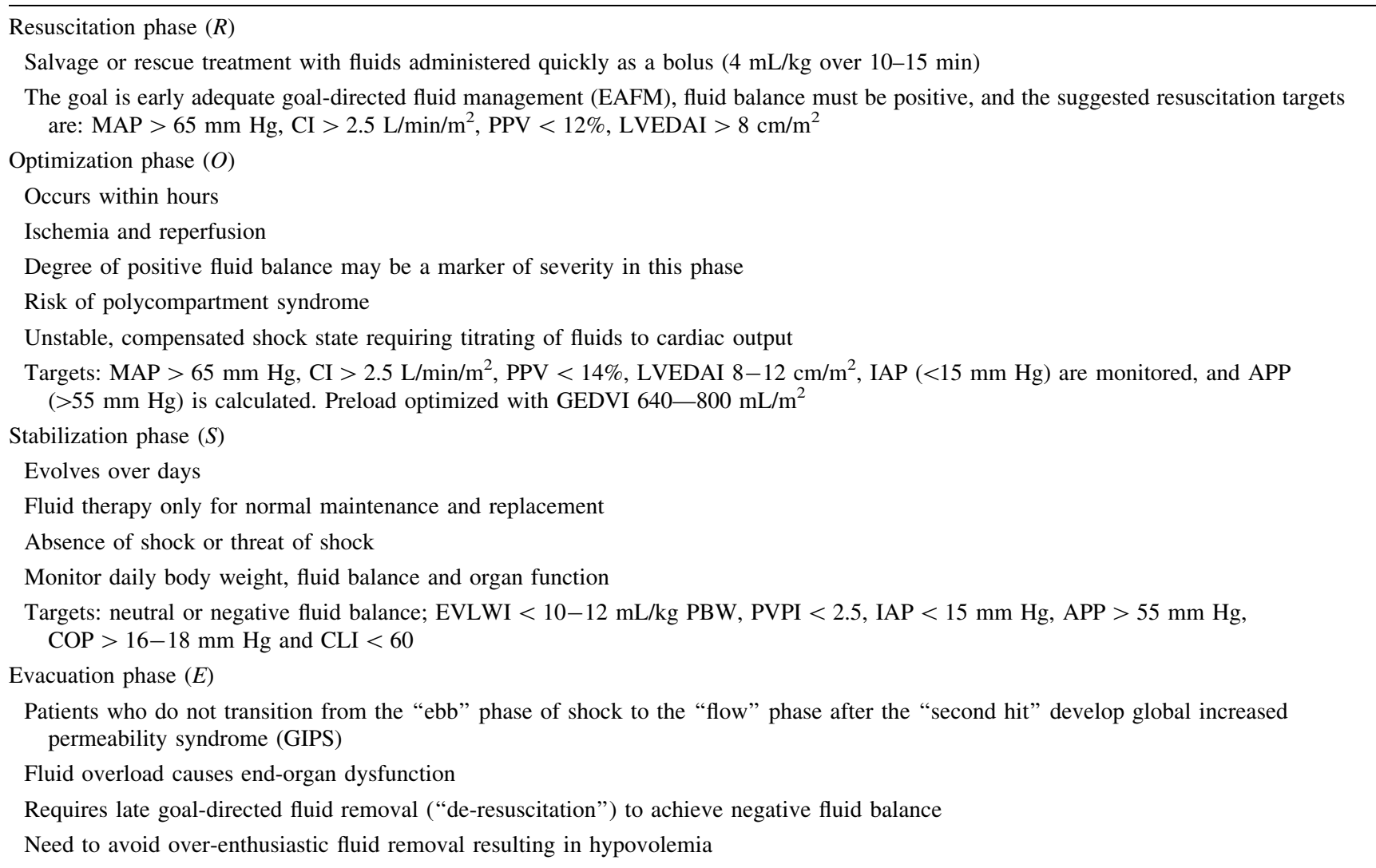

expands this compartment for longer. However, the design of the recent large studies involving HES showed that ongoing use of these fluids in critically ill patients, beyond initial resuscitation (even in the trauma subgroup analysis), was without benefit and may increase the need for renal replacement therapy [47].

During the post-resuscitation phase, crystalloids are not only required for fluid supplementation, but also as vehicles for administration of medication, including antibiotics, sedation and inotropes/vasopressors. The fluid required for the administration of these solutions together with those required for nutrition should as a guide not exceed $2 \mathrm{ml} / \mathrm{kg} /$ h. $0.9 \%$ "normal" saline has often been the fluid of choice for this purpose; however, concerns about both the sodium and chloride load may favor other "balanced" fluids $[13,50,51]$. This fluid can again be substituted for the one designed specifically for maintenance of daily fluid and electrolyte requirements once certainty regarding fluid requirements and responsiveness has been reached. During this period, the solutions infused as medications may also be made more concentrated to limit volume requirements.

It must be remembered that intravenous fluids are drugs providing both electrolytes and water. While calculating these requirements, the patient's medication and feed also need to be incorporated to avoid excessive volumes of each.

\section{Assessing volume status}

\section{Fluid responsiveness}

Only half of the ICU patients with hemodynamic instability are able to "respond" to fluid loading, which is explained by the shape of the Frank-Starling curve [52]. On the initial and steep limb of the curve, the stroke volume is highly dependent on preload: Administering fluid will actually result in a significant increase in stroke volume. In contrast, if the heart is working on the terminal and flat portion of the Frank-Starling curve, it cannot utilize any preload reserve and fluid administration will not significantly increase stroke volume. Accordingly, predictors of volume responsiveness are mandatory to distinguish between patients who can benefit from fluid and those in whom fluid is useless and hence deleterious.

\section{Static markers of cardiac preload}

Considering the Frank-Starling relationship, the response to volume infusion is more likely to occur when 
the ventricular preload is low, rather than when it is high. Unfortunately, none of the measures of cardiac preload enables to accurately predict fluid responsiveness: Neither the central venous pressure (CVP), the pulmonary artery occlusion pressure (PAOP), nor the left ventricular enddiastolic area (LVEDA) can discriminate between responders and non-responders to fluid therapy [24, 53, 54]. Only the right ventricular and the global end-diastolic volume have been proven to be of some benefit compared to barometric preload indicators especially in patients with increased intra-thoracic or intra-abdominal pressures $[23,55]$.

\section{Dynamic markers of volume responsiveness}

The alternative method for predicting volume responsiveness is simply to induce a change in cardiac preload and to observe the resulting effects on stroke volume or cardiac output or any available surrogate, i.e., to perform a "functional assessment" of the cardiac function [24]. This is achieved with intravenous fluid boluses [56]. This method can be criticized because repeated infusions of such amounts could eventually exert adverse effects if there is no preload reserve, especially if pulmonary permeability was increased.

\section{The respiratory variation of hemodynamic signals}

Observing the respiratory variation of hemodynamic signals has emerged as an alternative for assessing volume responsiveness without administering fluid. The concept is based on the assumption that the cyclic changes in right ventricular preload induced by mechanical ventilation should result in greater cyclic changes in left ventricular stroke volume when both ventricles operate on the steep rather than on the flat portion of the Frank-Starling curve, i.e., in case of biventricular preload preserve.

Numerous studies have consistently demonstrated that the magnitude of respiratory variation of surrogates of stroke volume allows predicting fluid responsiveness with accuracy [24]. Pulse pressure variation (PPV) is the most popular index, since it needs only an arterial catheter to be obtained and numerous bedside monitors calculate and display its value in real time. Reliability of PPV to predict fluid responsiveness has been demonstrated in ICU patients when it is calculated from a simple arterial catheter [4] or automatically calculated by simple bedside monitors such as the IntelliVue (Philips, USA) [5], the PiCCO (PULSION Medical Systems SE, Germany) [6] and the LiDCOplus (LiDCO Group PLC, UK) [7] monitors [57-61]. PPV can also be automatically obtained with the LiDCOrapid (LiDCO Group PLC, UK), Mostcare (Vytech, Italy) and Pulsioflex (MAQUET, Germany) uncalibrated monitors [62]. Noninvasive finger pressure monitors such as the
CNAP (CNSystems Medizintechnik AG, Austria) or ClearSight (Edwards Lifesciences Corporation, USA) also allow calculation of noninvasive PPV [63, 64]. It must be noted that this modality is difficult to use in several cases of trauma resuscitation as it requires controlled ventilation (i.e., no spontaneous ventilatory efforts), regular sinus rhythm and tidal volumes of $>7 \mathrm{ml} / \mathrm{kg}$.

\section{Other markers}

The following other surrogates of stroke volume respiratory variation can be used at the bedside:

- Respiratory variation of the pulse contour-derived stroke volume measured by the PiCCO or by the FloTrac/Vigileo (Edwards Lifesciences Corporation, USA) or by the LiDCOplus [58, 65, 66].

- Respiratory variation of the subaortic flow assessed by echocardiography and respiratory variation of the descending aortic blood flow assessed by esophageal Doppler [30, 67, 68].

- Other heart-lung interaction indices like respiratory variation of inferior or superior vena cava diameter (echocardiography), although limitations exist $[24,68-70]$.

- The passive leg raise (PLR) test carries an excellent ability to serve as a test of preload responsiveness, demonstrated in patients with acute circulatory failure $[71,72]$. A $10-12 \%$ increase in cardiac output or stroke volume during PLR enables prediction of fluid responsiveness, even patients with cardiac arrhythmias and/or spontaneous ventilator triggering [73]. However, in conditions of increased IAP and pain, the PLR may result in a false negative $[55,74]$.

- The end-expiratory occlusion test. The end-expiratory occlusion test can be used in patients with low lung compliance $[64,75]$.

- Ultrasonography: This bedside modality has advantages of repeatability, being noninvasive, with the ability to assess dynamic changes in the inferior vena cava (IVC) diameter, left ventricular outflow tract stroke volume variation, and estimate cardiac ejection fraction [76]. This provides the ability for real-time guidance of fluid resuscitation [77]. The most widely used method for assessing fluid responsiveness using IVC parameters is the caval index [78]. This measurement is most useful at extremes of volume status and is influenced by increases in ventilation parameters (tidal volume and positive end-expiratory pressure) and intra-abdominal pressure. As a result, despite positive findings in early studies, research has demonstrated a limited ability to detect those patients that would respond to further fluid resuscitation due to changes in these ventilatory 
parameters, and other patient factors such as obesity $[79,80]$. Other measurements using ultrasonography are possible such as SVV using pulse-wave Doppler, and aortic blood flow velocity using trans-esophageal echocardiography, but require more experience and may prove challenging in the emergency setting [81-83]. The combination of using ultrasonography to measure aortic velocity-time integral and combining this with a PLR test may be the best technique in skilled hands $[84,85]$.

Recommendations for current best practices:

1. If ultrasonography is available, then we advise using dynamic changes in IVC together with other clinical parameters. Preferably, if the skills are available, combining aortic velocity-time integral with dynamic changes from a PLR test should be performed.

2. In the absence of ultrasonography, dynamic markers of volume resuscitation should be attempted such as a PLR test, although this may have limited utility in the emergency setting due to patient injuries and pain.

3. If it is unable to utilize ultrasonography or PLR testing, other dynamic markers of fluid responsiveness can be attempted although these may be impractical in the emergency setting. Repeated "mini-boluses" of intravenous fluid (100-250 ml) can be used, provided that vigilance regarding excessive or inappropriate clear fluid administration is maintained.

\section{Special groups}

\section{Pediatrics}

Infants and children suffer from trauma, particularly vehicular trauma, with an increasing incidence in the developing world [86]. Children differ from adults in having a larger circulating blood volume ( $80 \mathrm{ml} / \mathrm{kg}$ in a term neonate) that decreases with age to the adult level of $70 \mathrm{ml} / \mathrm{kg}$ [87]. While the relative blood volume is larger, the absolute volumes required are small and should ideally be delivered through flow control devices (infusion pumps/syringe drivers) [88]. A cheap precaution is to deliver all clear fluids through a 60-drop-per-minute administration set rather than the 10-/ 15-drop-per-minute sets used for adults.

Restoration of circulating volume is a priority with the establishment of vascular access via a peripheral line, central (commonly femoral) line or intra-osseous line. The access should not be established distal to a site of injury (e.g., femoral line with blunt abdominal trauma) as the resuscitation fluid will extravasate into the injured area [89].
The principles of goal-directed therapy apply equally well to children as to adults. Initial resuscitation should be with $20 \mathrm{ml} / \mathrm{kg}$ of balanced crystalloid [90]. There is limited information on the efficacy and safety of synthetic colloids (e.g., HES) in children, with some evidence that hemodynamic goals are achieved more quickly and with smaller volumes but at increased cost and with no evidence of outcome benefit [91].

The role of all clear fluids is limited in trauma resuscitation due to their adverse effects of dilutional coagulopathy and anemia and generation of edema that hinders tissue perfusion and promotes organ dysfunction (including ileus, abdominal compartment syndrome and ARDS). The volume of clear fluid should not exceed $40 \mathrm{ml} / \mathrm{kg}$ [90]. Administration of blood and blood products (platelets and plasma) should be considered depending on the response to the initial $20 \mathrm{ml} / \mathrm{kg}$ crystalloid bolus and the severity of injury. Due to the small volumes required, many pediatricians use human colloids such as plasma or albumin for intravascular volume replacement in preference to synthetic clear fluids [92].

During the maintenance phase of resuscitation, children appear to be at risk of hyponatremia. This seems to be due to administration of excessive volumes of hypotonic solutions such as $1 / 2$ strength Darrow's solution (Na-61, K-12, Cl-52, Lactate- $27 \mathrm{mmol} / \mathrm{l}$ ) with $5 \%$ dextrose resulting in hyponatremia and potentially fatal cerebral edema [93]. A clear distinction is required between resuscitation fluids, that must be isotonic and preferably balanced, and maintenance fluids, that may be hypotonic and should only be given in limited volumes (maximum $2 \mathrm{ml} / \mathrm{kg} / \mathrm{h}$ ) via a flow controller to prevent rapid administration [94].

\section{Elderly}

The World Health Organization defines "elderly" as a chronological age of 65 years or more. Age is only one criterion in the assessment of overall health leading to the concept of biological age based on organ dysfunction and/ or chronic disease [95]. Despite advances in trauma care, the elderly, either chronologically or biologically, are at increased risk of morbidity, particularly limitation of mobility and self-care ability, and mortality after trauma [96].

Cardiovascular changes of aging include stiffening of the arterial circulation and loss of compliance of the left ventricle. The elderly thus tolerate hypo- and hypervolemia poorly. Volume loss reduces preload resulting in ventricular under-filling and a disproportionate drop in cardiac output. Over-hydration is as dangerous due to the lack of ventricular compliance predisposing to the development of edema, particularly pulmonary edema [97]. Assessment of fluid requirements in the elderly is best done by 
echocardiography as noninvasive measurements based on pressure or pulse contour analysis are subject to variation due to the changes in the cardiovascular system from aging [98].

Clear fluid administration should initially be limited to $20 \mathrm{ml} / \mathrm{kg}$ with early consideration given to the administration of blood and blood products. Careful re-evaluation and vigilant monitoring should to performed to determine if further fluid administration is required; particularly if underlying heart disease is suspected. Due to the likelihood of underlying coronary and cerebral artery disease in the elderly, consideration should be given to maintaining hemoglobin levels above $9 \mathrm{~g} / \mathrm{dl}$ and mean arterial pressure above $70 \mathrm{mmHg}$, particularly if the patients comorbidities are unknown [97].

\section{Burns}

Appropriate and effective initial resuscitation of victims of burns is vital for survival and reduction of morbidity and mortality [99]. The deeper and more extensive the burn, the greater the fluid requirements, but excessive fluid administration will also increase morbidity by generation of edema [100]. The formulas used for calculating volume requirements (e.g., Brooke \& Parkland) use only body surface area (BSA) and do not compensate for depth. In clinical practice, the fluid requirement is approximately $5 \mathrm{ml} / \mathrm{kg} / \%$ BSA during the first $24 \mathrm{~h}$ [101].

The rate of fluid administration will initially be rapid with up to half the daily requirement given in the first $6 \mathrm{~h}$. The use of colloid solutions is controversial. Hyperoncotic colloids worsen outcome, but the role of albumin or synthetic colloids (e.g., HES) is less clear [102-104]. Colloid solutions shorten the time to achieve hemodynamic goals, but increase expense without a concomitant improvement in outcome.

Enteral resuscitation is effective if commenced within $6 \mathrm{~h}$. Placement of a feeding tube should be part of the resuscitation protocol for burns. Delay of feeding for more than $6 \mathrm{~h}$ will result in an increasing feeding failure due to gastroparesis and ileus. Maintenance of enteral feeding maintains the gut associated lymphoid tissue that participates in maintenance of immunity at all epithelial surfaces including the skin. A standard formula may be used starting at $2 \mathrm{ml} / \mathrm{kg} / \mathrm{h}$ and increasing incrementally every $3 \mathrm{~h}$ until the goal rate calculated for each patient is reached [105].

Two simple investigations should be used to monitor the effectiveness of resuscitation from burns. The first is the hematocrit, which may be as high as $70 \%$ on admission after extensive burns. Failure to reduce the hematocrit below $40 \%$ within the first $6 \mathrm{~h}$ is an accurate indicator of poor prognosis. The second is urine output, which should be maintained at around $1 \mathrm{ml} / \mathrm{kg} / \mathrm{h}$. Development of acute renal failure carries a very poor prognosis with extensive burns [106]. A more detailed review of fluids in burn resuscitation is beyond the scope of this paper; however, further references are provided [107, 108].

\section{Crush injury/syndrome}

Crush injury is seen in victims of motor vehicle collisions who are entrapped and have limbs compressed, resulting in direct muscle trauma followed by a reperfusion injury when freed. Similar injury is seen in prolonged immobilization (after a fall or drug overdose) and entrapment in collapsed buildings after natural disasters [109]. South Africa has an unfortunate history of interpersonal violence. With the breakdown in the rule of law in many communities, alleged criminals may be assaulted by community members using traditional whips (sjamboks). This results in extensive muscle injury; however, muscle perfusion is maintained so reperfusion does not occur [110]. Muscle injury releases myoglobin that is detrimental to kidneys. A surrogate marker for myoglobin is creatine kinase $(\mathrm{CK})$ used as follows (Table 3).

With diffuse injury, such as community sjambok assaults, the surface area of the body injured should be quantified as for burns. A surface area of $>18 \%$ carries increased risk of renal dysfunction [110]. Aggressive fluid loading (20-40 ml/kg initial bolus followed by $10-20 \mathrm{ml} /$ $\mathrm{kg} / \mathrm{h}$ ) should begin as soon as the patient makes contact with the healthcare system. In the pre-hospital environment, fluid loading should ideally occur prior to release of crushed limb/s [111].

Traditionally, $0.9 \%$ saline is used for fluid loading. Alternatives, to limit the occurrence of hypernatremia and hyperchloremic acidosis, include $0.45 \%$ saline and alternating $0.9 \%$ saline and $5 \%$ dextrose. Balanced solutions such as modified Ringer's lactate are not recommended due to concerns regarding hyperkalemia, but this risk may be offset by the hyperchloremic acidosis seen with largevolume saline administration [109].

Should presentation be delayed, creatinine and potassium should be measured while initial fluid loading occurs, as the kidneys may have been damaged beyond immediate recovery. Failure to produce urine after initial fluid loading associated with an elevated urea, creatinine and potassium

Table 3 Representation of how to use creatine kinase as a surrogate marker for myoglobin

\begin{tabular}{lll}
\hline CK U/1 & Risk of renal failure & Admission \\
\hline$<500$ & Low & Unlikely \\
$500-5000$ & Intermediate & At least overnight \\
$>5000 \mathrm{U} / 1$ & High & Admission to ICU/high care \\
\hline
\end{tabular}


indicates the need for urgent renal replacement therapy. Further fluid loading should not be administered, as the absolute volume overload that arises in the absence of urine output will result in pulmonary edema with hypoxia requiring intubation and ventilation. There is no role for loop or osmotic diuretics, and the use of sodium bicarbonate to induce alkaline diuresis is also not supported by evidence [110].

\section{Pregnancy}

Trauma, particularly vehicular, or due to intimate partner violence is a common cause for maternal and fetal morbidity and mortality. In the developing world, neonatal intensive-care facilities are limited so maternal considerations take precedence in resuscitation until fetal viability is likely [112].

Pregnancy duration of more than 20 weeks makes aortocaval compression a realistic cause of hypotension during resuscitation, emphasizing the need for maintaining a $20^{\circ}$ left lateral tilt [113]. Fluid administration follows accepted principles of resuscitation [112].

Should delivery occur during resuscitation, significant blood loss may occur due to post-partum hemorrhage. Oxytocin availability may be limited due to expense and requirement for refrigeration. Misoprostol is an accepted alternative, but is only available in an oral form that may need to be administered rectally during resuscitation [114]. It should be remembered that the physiological compensation for blood loss might be better tolerated in pregnancy due to the physiological changes that predominantly take place in the second and third trimesters and include an increased circulating blood volume and cardiac output. Awareness of this should be maintained to avoid underestimation of blood loss and underlying injuries.

\section{Conclusion}

Fluids are drugs and should be managed as such. Appropriate early fluid resuscitation in trauma patients is a challenging task. Care should be taken in selecting both the type and volume to promote appropriate perfusion and oxygen delivery, avoiding the adverse effects seen when giving too little or too much. Ongoing fluid strategies following resuscitation should incorporate dynamic markers of volume status whenever possible. All aspects of fluid administration should be incorporated into daily fluid plans, including feeding and infusions of medications. A sound knowledge of the differences and physiological consequences of specific trauma groups is essential for all practitioners delivering care for trauma patients [7].
Acknowledgements The costs covering the open access publication (USD3000) of this review article were covered by an unrestricted educational grant from the International Fluid Academy (IFA). The IFA is integrated within the not-for-profit charitable organization iMERiT (International Medical Education and Research Initiative) under Belgian Law. The IFA website (http://www.fluidacademy.org) is now an official SMACC (Social Media and Critical Care) affiliated site and its content is based on the philosophy of FOAM (Free Open Access Medical Education - \#FOAMed).

\section{Compliance with ethical standards}

Competing interest Robert Wise and Michael Faurie declare that they have no competing interests. Manu LNG Malbrain is member of the executive committee of the International Fluid Academy (IFA). Eric Hodgson is a paid speaker and advisory board member for Fresenius-Kabi.

Open Access This article is distributed under the terms of the Creative Commons Attribution 4.0 International License (http://crea tivecommons.org/licenses/by/4.0/), which permits unrestricted use, distribution, and reproduction in any medium, provided you give appropriate credit to the original author(s) and the source, provide a link to the Creative Commons license, and indicate if changes were made.

\section{References}

1. Strunden M, Heckel K, Goetz A et al (2011) Perioperative fluid and volume management: physiological basis, tools and strategies. Ann Intensive Care 1:1-8

2. Marik P (2014) Iatrogenic salt water drowning and the hazards of a high central venous pressure. Ann Intensive Care 4:1-9

3. Chappell D, Jacob M, Hofmann-Kiefer K et al (2008) A rational approach to perioperative fluid management. Anesthesiology 109:723-740

4. Myburgh J, Finfer S, Bellomo R et al (2012) Hyrdoxyethyl starch or saline for fluid resuscitation in intensive care. $\mathrm{N}$ Engl $\mathrm{J}$ Med 367:1901-1911

5. Perner A, Nicolai H, Guttormson A et al (2012) Hydroxyethyl starch 130/0.42 versus Ringer's acetate in severe sepsis. N Engl J Med 367:124-134

6. Raiman M, Mitchell C, Biccard B et al (2016) Comparison of hydroxyethyl starch colloids with crystalloids for surgical patients: a systematic review and meta-analysis. Eur J Anaesthesiol 33(42-4):8

7. Annane D, Siami S, Jaber S et al (2013) Effects of fluid resuscitation with colloids vs crystalloids on mortality in critically ill patients presenting with hypovolaemic shock. The CRISTAL randomized trial. JAMA 310(1809-181):7

8. Chappell D, Jacob M (2013) Hydroxyethyl starch-the importance of being earnest. Scand J Trauma Resusc Emerg Med 21:1-4

9. Chappell D, Jacob M (2013) Twisting and ignoring facts on hydroxyethyl starch is not very helpful. Scand J Trauma Resusc Emerg Med 21:1-3

10. Coppola S, Froio S, Chiumello D (2014) Fluid resuscitation in trauma patients: what should we know? Curr Opin Crit Care 20:444-450

11. Doig G, Heighes P, Simpson F et al (2011) Early enteral nutrition reduces mortality in trauma patients requiring intensive care: a meta-analysis of randomised controlled trials. Injury 42(50-5):6 
12. Guidet B, Martinet O, Boulain T et al (2012) Assessment of hemodynamic efficacy and safety of 6\% hydroxyethylstarch $130 / 0.4$ vs $0.9 \% \mathrm{NaCl}$ fluid replacement in patients with severe sepsis: the CRYSTMAS study. Crit Care 16(3):R94

13. Krajewski M, Raghunathan K, Paluszkiewicz S et al (2015) Meta-analysis of high- versus low-chloride content in perioperative and critical care fluid resuscitation. Br J Surg 102:24-36

14. Ince C, Groeneveld A (2014) The case for $0.9 \% \mathrm{NaCl}$ : is the undefendable, defensible? Kidney Int 86:1087-1095

15. Lira A, Pinsky M (2014) Choices in fluid type and volume during resuscitation: impact on patient outcomes. Ann Intensive Care 4:38

16. Martini W, Cortez D, Dubick M (2013) Comparisons of normal saline and lactated Ringer's resuscitation on hemodynamics, metabolic responses, and coagulation in pigs after severe hemorrhagic shock. Scand J Trauma Resusc Emerg Med 21:86

17. Hafizah M, Liu C, Ooi J (2015) Normal saline versus balancedsalt solution as intravenous fluid therapy during neurosurgery: effects on acid-base balance and electrolytes. J Neurosurg Sci. [Epub ahead of print]

18. Galvagno S, Mackenzie C (2013) New and future resuscitation fluids for trauma patients using hemoglobin and hypertonic saline. Anaesthesiol Clin 31:1-19

19. Patanwala A, Amini A, Erstad B (2010) Use of hypertonic saline injection in trauma. Am J Health Syst Pharm 67:1920-1928

20. Finfer S, Bellomo R, Boyce N et al (2004) A comparison of albumin and saline for fluid resuscitation in the intensive care unit. N Engl J Med 350:2247-2256

21. Myburgh J, Cooper D, Finfer S et al (2007) Saline or albumin for fluid resuscitation in patients with traumatic brain injury. N Engl J Med 357:874-884

22. Brackney C, Diaz L, Milbrandt E et al (2010) Is albumin use SAFE in patients with traumatic brain injury? Crit Care 14:307

23. Malbrain M, Roberts D, Sugrue M et al (2014) The polycompartment syndrome: a concise state-of-the-art review. Anaesthesiol Intensive Ther 46:433-450

24. Marik P, Monnet X, Teboul J (2011) Hemodynamic parameters to guide fluid therapy. Ann Intensive Care 1:1-9

25. Hahn R, Bergek C, Geback T et al (2013) Interactions between the volume effects of hydroxyethyl starch 130/0.4 and Ringer's acetate. Crit Care 17:R104

26. Strunden M, Heckel K, Goetz A et al (2011) Perioperative fluid and volume management: physiological basis, tools and strategies. Ann Intensive Care 1(1):2

27. Spinella P, Perkin J, Grathwohl K et al (2009) Warm fresh whole blood is independently associated with improved survival for patients with combat-related traumatic injuries. J Trauma 66:S69-S76

28. Raymer J, Flynn L, Martin R (2012) Massive transfusion of blood in the surgical patient. Surg Clin N Am 92:221-234

29. Holcomb J, Tilley B, Baraniuk S et al (2015) Transfusion of plasma, platelets and red blood cells in a $1: 1: 1$ vs a $1: 1: 2$ ratio and mortality in patients with severe trauma: the PROPPR randomised controlled trial. JAMA 313:471-482

30. Monnet X, Rienzo M, Osman D et al (2005) Esophageal Doppler monitoring predicts fluid responsiveness in critically ill ventilated patients. Intensive Care Med 31:1195-1201

31. Holcomb J (2010) Optimal use of blood products in severely injured trauma patients haematology. Am Soc Hematol Educ Progr 1:465-469

32. Miller T (2013) New evidence in trauma resuscitation-is $1: 1: 1$ the answer? Perioper Med 2(1):13

33. Network for the advancement of patient blood management, haemostasis, and thrombosis - Haemoglobin based solutions (2016). www.nataonline.com/np/421/hemoglobin-based-solutions
34. Levien L (2006) South Africa: clinical experience with hemopure. ISBT Sci Ser 1:167-173

35. MacKenzie C, Pitman A, Hodgson R et al (2015) Are hemoglobin-based oxygen carriers being withheld because of regulatory requirement for equivalence to packed red blood cells? Am J Ther 22:e115-e121

36. Natanson C, Kern S, Lurie P et al (2008) Cell-free hemoglobinbased blood substitutes and risk of myocardial infarction and death: a meta-analysis. JAMA 299:2304-2312

37. Africa SS (2015) Mid-year population estimates 2015. Statistical release P0302; Pretoria, South Africa. http://www.statssa. gov.za/?page_id=1854\&PPN=P0302\&SCH=6334

38. van Ramshorst GH, Salih M, Hop WC et al (2011) Noninvasive assessment of intra-abdominal pressure by measurement of abdominal wall tension. J Surg Res 171:240-244

39. Gonzalez E, Moore E, Moore H et al (2016) Goal-directed hemostatic resuscitation of trauma-induced coagulopathy: a Pragmatic randomized clinical trial comparing a viscoelastic assay to conventional coagulation assays. Ann Surg 263:1051-1059

40. Tapia N, Chang A, Norman M (2012) TEG-guided resuscitations superior to standardized MTP resuscitation massively transfused penetrating trauma patients. J Acute Care Surg 74:378-386

41. Haas T, Gorlinger K, Grassetto A et al (2014) Thromboelastometry for guiding bleeding management of the critically ill patient: a systematic review of the literature. Min Anestesiol 80:1320-1335

42. Adhikary S, Pruett A, Thiruvenkatarajan V (2014) Coagulation testing in the perioperative period. Indian J Anaesth 58:565-572

43. Bellamy M (2006) Wet, dry or something else? Br J Anaesth 97:755-757

44. Malbrain M, Marik P, Witters I et al (2014) Fluid overload, deresuscitation, and outcomes in critically ill or injured patients: a systematic review with suggestions for clinical practice. Anaesthesiol Intensive Ther 46:361-380

45. Shere-Wolfe R, Galvagno SJ, Grissom T (2012) Critical care considerations in the management of the trauma patient following initial resuscitation. Scand J Trauma Resusc Emerg Med 20:1-15

46. Teboul J, Monnet X (2009) Detecting volume responsiveness and unresponsiveness in intensive care unit patients: two different problems, only one solution. Crit Care 13:R111

47. Malbrain M, Van Regenmortel N, Owczuk R (2014) The debate on fluid management and haemodynamic monitoring continues: between Scylla and Charybdis, or faith and evidence. Anaesthesiol Intensive Ther 46:313-318

48. Malbrain ML, Van Regenmortel N, Owczuk R (2015) It is time to consider the four D's of fluid management. Anaesthesiol Intensive Ther 47:1-5

49. Van Regenmortel N, Jorens PG, Malbrain ML (2014) Fluid management before, during and after elective surgery. Curr Opin Crit Care 20:390-396

50. Langer T, Santini A, Scotti E et al (2015) Intravenous balanced solutions: from physiology to clinical evidence. Anaesthesiol Intensive Ther 47:s78-s88

51. Finfer S, Liu B, Taylor C et al (2010) Resuscitation fluid use in critically ill adults: an international cross-sectional study in 391 intensive care units. Crit Care 14:R185

52. Michard F, Teboul J (2002) Predicting fluid responsiveness in ICU patients: a critical analysis of the evidence. Chest 121:2000-2008

53. Marik P, Baram M, Vahid B (2008) Does the central venous pressure predict fluid responsiveness? A systematic review of the literature and the tale of seven mares. Chest 134:172-178 
54. Marik P, Cavallazzi R (2013) Does the central venous pressure predict fluid responsiveness? An updated meta-analysis and a plea for some common sense. Crit Care Med 41:1774-1781

55. Malbrain M, De Waele J, De Keulenaer B (2015) What every ICU clinician needs to know about the cardiovascular effects caused by abdominal hypertension. Anaesthesiol Intensive Ther 47:388-399

56. Vincent J, Weil M (2006) Fluid challenge revisited. Crit Care Med 34:1333-1337

57. Cannesson M, Slieker J, Desebbe O et al (2008) The ability of a novel algorithm for automatic estimation of the respiratory variations in arterial pulse pressure to monitor fluid responsiveness in the operating room. Anesth Analg 106:1195-1200

58. Cecconi M, Monti G, Hamilton M et al (2012) Efficacy of functional hemodynamic parameters in predicting fluid responsiveness with pulse power analysis in surgical patients. Min Anestesiol 78:527-533

59. Hofer C, Müller S, Furrer L et al (2005) Stroke volume and pulse pressure variation for prediction of fluid responsiveness in patients undergoing off-pump coronary artery bypass grafting. Chest 128:848-854

60. Michard F, Boussat S, Chemla D et al (2000) Relation between respiratory changes in arterial pulse pressure and fluid responsiveness in septic patients with acute circulatory failure. Am J Respir Crit Care Med 162:134-138

61. Peeters Y, Bernards J, Mekeirele M et al (2015) Hemodynamic monitoring: to calibrate or not to calibrate? Part $1-$ calibrated techniques. Anaesthesiol Intensive Ther 47:487-500

62. Bernards J, Mekeirele M, Hoffmann B et al (2015) Hemodynamic monitoring: to calibrate or not to calibrate? Part 2non-calibrated techniques. Anaesthesiol Intensive Ther 47: 501-516

63. Ameloot K, Palmers P, Malbrain M (2015) The accuracy of noninvasive cardiac output and pressure measurements with finger cuff: a concise review. Curr Opin Crit Care 21:232-239

64. Monnet X, Dres M, Ferré A et al (2012) Prediction of fluid responsiveness by a continuous non-invasive assessment of arterial pressure in critically ill patients: comparison with four other dynamic indices. Br J Anaesth 109:330-338

65. Berkenstadt H, Margalit N, Hadani M et al (2001) Stroke volume variation as a predictor of fluid responsiveness in patients undergoing brain surgery. Anesth Analg 92:984-989

66. Biais M, Nouette-Gaulain K, Cottenceau V et al (2008) Uncalibrated pulse contour-derived stroke volume variation predicts fluid responsiveness in mechanically ventilated patients undergoing liver transplantation. Br J Anaesth 101:761-768

67. Feissel M, Michard F, Mangin I et al (2001) Respiratory changes in aortic blood velocity as an indicator of fluid responsiveness in ventilated patients with septic shock. Chest 119:867-873

68. Vermeiren G, Malbrain M, Walpot J (2015) Cardiac ultrasonography in the critical care setting: a practical approach to asses cardiac function and preload for the "non-cardiologist". Anaesthesiol Intensive Ther 47:89-104

69. Feissel M, Michard F, Faller J et al (2004) The respiratory variation in inferior vena cava diameter as a guide to fluid therapy. Intensive Care Med 30:1834-1837

70. Vieillard-Baron A, Chergui K, Rabiller A et al (2004) Superior vena caval collapsibility as a gauge of volume status in ventilated septic patients. Intensive Care Med 30:1734-1739

71. Cavallaro F, Sandroni C, Marano C et al (2010) Diagnostic accuracy of passive leg raising for prediction of fluid responsiveness in adults: systematic review and meta-analysis of clinical studies. Intensive Care Med 36:1475-1483

72. Monnet X, Teboul J (2008) Passive leg raising. Intensive Care Med 34:659-663
73. Monnet X, Rienzo M, Osman D et al (2006) Passive leg raising predicts fluid responsiveness in the critically ill. Crit Care Med 34:1402-1407

74. Malbrain M (2010) Reuter D Assessing fluid responsiveness with the passive leg raising maneuver in patients with increased intra-abdominal pressure: be aware that not all blood returns! Crit Care Med 38:1912-1915

75. Monnet X, Osman D, Ridel C et al (2009) Predicting volume responsiveness by using the end-expiratory occlusion in mechanically ventilated intensive care unit patients. Crit Care Med 37:951-956

76. Farcy D, Jain A, Dalley M et al (2016) Pitfalls in using central venous pressure as a marker of fluid responsiveness. Emerg Med 48(1):18-28

77. Zhang Z, Xu X, Ye S et al (2014) Ultrasonographic measurement of the respiratory variation in the inferior vena cava diameter is predictive of fluid responsiveness in critically ill patients: systematic review and meta-analysis. Ultrasound Med Biol 40:845-853

78. Nagdev A, Merchant R, Tirado-Gonzalez A et al (2010) Emergency department bedside ultrasonographic measurement of the caval index for noninvasive determination of low central venous pressure. Ann Emerg Med 55:290-295

79. Corl K, Napoli A, Gardiner F (2012) Bedside sonographic measurement of the inferior vena cava caval index is a poor predictor of fluid responsiveness in emergency department patients. Emerg Med Aust 24:534-539

80. Muller L, Bobbia X, Toumi M et al (2012) Respiratory variations of inferior vena cava diameter to predict fluid responsiveness in spontaneously breathing patients with acute circulatory failure: need for a cautious use. Crit Care 16(5):R188

81. Chin J, Jun I, Lee J et al (2014) Can stroke volume variation be an alternative to central venous pressure in patients undergoing kidney transplantation? Transplant Proc 46:3363-3366

82. Zhang Z, Lu B, Sheng X et al (2011) Accuracy of stroke volume variation in predicting fluid responsiveness: a systematic review and meta-analysis. J Anesth 25:904-916

83. Feissel M, Michard F, Mangin I et al (2001) Respiratory changes in aortic blood velocity as an indicator of fluid responsiveness in ventilated patients with septic shock. Chest 119:867-873

84. Lamia B, Ochagavia A, Monnet X et al (2007) Echocardiographic prediction of volume responsiveness in critically ill patients with spontaneously breathing activity. Intensive Care Med 33:1125-1132

85. Maizel J, Airapetian N, Lorne E et al (2007) Diagnosis of central hypovolemia by using passive leg raising. Intensive Care Med 33(7):1133-1138

86. Möller H, Falster K, Ivers R et al (2015) Inequalities in unintentional injuries between indigenous and non-indigenous children: a systematic review. Inj Prev 21:e144-e152

87. Lacroix J, Demaret P, Tucci M (2012) Red blood cell transfusion: decision making in pediatric intensive care units. Semin Perinatol 36:225-231

88. Murat I, Dubois M (2008) Perioperative fluid therapy in paediatrics. Ped Anesth 18:363-370

89. de Caen A, Reis A, Bhutta A (2008) Vascular access and drug therapy in pediatric resuscitation. Pediatr Clin N Am 55:909-927

90. Hussmann B, Lefering R, Kauther M et al (2012) Influence of prehospital volume replacement on outcome in polytraumatized children. Crit Care 16:R201

91. Sömpelmann R, Kretz F-J, Luntzer R et al (2012) Hydroxyethyl starch 130/0.42/6:1 for perioperative plasma volume replacement in 1130 children: results of an European prospective multicenter observational postauthorization safety study (PASS). Ped Anesth 22:371-378 
92. Dzik W, Kyeyune D, Otekat G et al (2015) Transfusion medicine in Sub-Saharan Africa: conference summary. Transfus Med Rev 29:195-204

93. Terris M, Crean P (2011) Fluid and electrolyte balance in children. Anaesth Intens Care Med 13:15-19

94. Au A (2010) Bell M Prevention of hospital-acquired hyponatremia in children: are hypotonic solutions safe? Pediatr Crit Care Med 11:528-529

95. Maguire S, Slater B (2010) Physiology of ageing. Anaesth Intensive Care Med 11:290-292

96. Stevens C, Torke A (2016) Geriatric trauma: a clinical and ethical review. J Trauma Nurs 23:36-41

97. Corcoran T, Hillyard S (2011) Cardiopulmonary aspects of anaesthesia for the elderly. Best Prac Res Clin Anaesthesiol 25:329-354

98. Marik P (2006) Management of the critically ill geriatric patient. Crit Care Med 34:S176-S182

99. Alvarado R, Chung K, Cancio L et al (2009) Burn resuscitation. Burns 35:4-14

100. Bishop S, Maguire S (2012) Anaesthesia and intensive care for major burns. Cont Ed Anaesth Crit Care Pain 12:118-122

101. Greenhalgh D (2010) Burn resuscitation: the results of the ISBI/ ABA survey. Burns 36:176-182

102. Béchir M, Puhan M, Neff S et al (2010) Early fluid resuscitation with hyperoncotic hydroxyethyl starch $200 / 0.5$ (10\%) in severe burn injury. Crit Care 14:R123

103. Cochran A, Morris S, Edelman L et al (2007) Burn patient characteristics and outcomes following resuscitation with albumin. Burns 33:25-30

104. Vlachou E, Gosling P, Moiemen N (2010) Hydroxyethylstarch supplementation in burn resuscitation-a prospective randomised controlled trial. Burns 36:984-991
105. Khorasani E, Mansouri F (2010) Effect of early enteral nutrition on morbidity and mortality in children with burns. Burns 36:1067-1071

106. Legrand M, Guttormsen A, Berger M (2015) Ten tips for managing critically ill burn patients: follow the RASTAFARI! Intensive Care Med 41:1107-1109

107. Peeters Y, Vandervelden S, Wise R et al (2015) An overview on fluid resuscitation and resuscitation endpoints in burns: past, present and future. Part 1-historical background, resuscitation fluid and adjunctive treatment. Anaesthesiol Intensive Ther 47:s6-s14

108. Peeters Y, Lebeer M, Wise R et al (2015) An overview on fluid resuscitation and resuscitation endpoints in burns: past, present and future. Part 2-avoiding complications by using the right endpoints with a new personalized protocolized approach. Anaesthesiol Intensive Ther 47:s15-s26

109. Jagodzinski N, Weerasingheand C, Porter K (2010) Crush injuries and crush syndrome-a review. Part 1: the systemic injury. Trauma 12:69-88

110. Hardcastle T, Smith W (2011) A crushing experience: the spectrum and outcome of soft tissue injury and myonephropathic syndrome at an Urban South African University Hospital. Af J Emerg Med 1:17-24

111. Wood D (2011) Rosedale K Crush Syndrome in the rural setting. Emerg Med J 28:817

112. Moon T, Sappenfield J (2016) Anesthetic management and challenges in the pregnant patient. Curr Anesthesiol Rep 6:89-94

113. Jeejeebhoy F, Morrison L (2013) Maternal cardiac arrest: a practical and comprehensive review. Emerg Med Int 2013:274814. doi: $10.1155 / 2013 / 274814$

114. Tunçalp Ö, Souza J, Gülmezoglu M (2013) New WHO recommendations on prevention and treatment of post-partum haemorrhage. Int J Gynecol Obstet 123:254-256 